\title{
Identification of Six novel missense single nucleotide polymorphisms in the MAOA gene predisposing to aggressive behavior. Bioinformatics study.
}

Abdelrahman H. Abdelmoenim ${ }^{1 *}$, Mujahed I. Mustafa ${ }^{2}$, Naseem S. Murshed ${ }^{3}$,Nosiba S. Omer ${ }^{4}$, Alaa I. Mohammed $^{5}$, Rania A. Abdulmajed ${ }^{6}$, Enas dk. Dawoud ${ }^{7}$, Abdelrafie M. Makhawi².

1-Faculty of medicine, Al-Neelain university, Khartoum, Sudan.

2-Department of Biotechnology, University of Bahri, Khartoum, Sudan.

3-Department of Bioinformatics, Africa City of Technology, Khartoum North, Sudan.

4-Faculty of nursing, Shandi university, Khartoum, Sudan.

5-Department of Hematology \& Immunohematology, Faculty of Medical Laboratory Sciences, University of Khartoum, Khartoum, Sudan.

6-M.D. Clinical Psychiatry resident,Sudan Medical Specialization Board,Khartoum, Sudan.

7-Faculty of Medical laboratory sciences, Razi University, Khartoum, Sudan.

*Corresponding author email: abduhamza009@gmail.com.

\begin{abstract}
:
Background: An astonishing observation is that aggressive behavior is actually a highly heritable. Recent experimental work and behavior research has linked individual variation in a functional polymorphism of the monoamine oxidase-A gene (MAOA) to the occurrence of anger-driven aggression. Aggressive antisocial and violent behavior has become a regularly debated topic in the scientific community; the impending question is what is the source of aggressive behavior, is it genetic or environmental or is it just an individual choice. This study aims to analyses the SNPs found in MAOA gene and it is possible association to aggressive behavior.

Method: Various bioinformatics software (SIFT, PolyPhen-2, PROVEAN, SNAP22, SNP\&GO and PMut)is used to analyses the SNPs within the MAOA gene to study the structural and functional implication on the associated protein, which is further clarified using chimera software. Then gene- gene interaction is studied with geneMANIA software. Furthermore, conservation and annotation studies were done through the ConSurf server and Variant Effect Predictor (VEP) respectively.
\end{abstract}


Result: Six missense SNPs were found to affect the structural and functional prospect of MAOA protein.

Conclusion:Genetic mutation within MAOA is likely to be associated with aggressive behavior; this will enrich future management and screening possibilities for this behavior.

Keywords: Aggressive behavior, Antisocial behavior, MAOA, SNPs, bioinformatics.

\section{Introduction:}

"Aggression is defined as harmful social interaction with the intention of inflicting damage or other unpleasantness upon another individual. It can happen with or without trigger(1). However, aggressive behavior is considered as an adaptation mechanism not only in humans but in other species as well. Furthermore, sometimes the aggressive response can be an indication of psychopathology especially when it exceeds the normal range (2).

Studies had shown that, Aggressive and antisocial behavior is highly heritable (3). When studying the heritability of aggression and antisocial behaviors the MAOA gene is usually the gene of interest. The effect of MAOA in modulating human behavior has been widely studied. Moreover, associations have been described between several psychiatric illnesses and altered MAO-A function. Even more, antisocial personality disorder (ASPD) and borderline personality disorder (BPD) have been the main focus of many studies regarding the MAO A gene in non-clinical samples (4).

a study done between 2000 and 2002 on a sample of 566 patients had concluded that the Genetic variants of the monoamine oxidase A (MAOA) have been associated with aggression-, anxiety-, and addiction-related behavior (5). Nevertheless, both main and interactive effects of the MAOA VNTR have been associated with ASPD and its symptomatology. It was noted that people with antisocial personality disorder usually manifest themselves as being irritable and aggressive, engaging in behavior that results in a criminal arrest,and they lack remorse for their actions(6).

Recent experimental work and behavior research has linked individual variation in a functional polymorphism of the monoamine oxidase-A gene (MAOA) to the occurrence of anger-driven aggression (7-10).

Furthermore, there is extensive research examining the relation between a polymorphism in the promoter region of the MAOA gene and antisocial phenotypes, as it has been found that 2-repeat allele-may has effects in modulating aggressive behaviors $(11,12)$. Historically the clinical condition caused by MAOA deficiency, namely Brunner syndrome, was one of the first disorders to be described in relation to MAOA gene and it is characterized by overt antisocial and aggressive conduct (13). studies have found that interaction of low MAOA transcription polymorphisms with environmental factors such as early abuse, deprivation and iron deficiency anemia has been found to increase the occurrence of violent antisocial behavior $(14,15)$. 
The MAO is a mitochondrial enzyme. MAO family composed of two isoenzymes, termed A (Genbank Acc. No: BC008064.1) and B (Genbank Acc. No: M69177), which despite a substantial structural overlap, are remarkably different for substrate preference, inhibitor selectivity, regional distribution, and functional role in behavioral regulation (16). The two monoamine oxidase (MAO) enzymes, $\mathrm{A}$ and $\mathrm{B}$, preferentially deaminatesmonoamine neurotransmitters, such as serotonin, norepinephrine, and dopamine. The genes encoding for both types are mapped on chromosome Xp11.23 (17).

It has been found that the termination of serotonin (5-hydroxytryptamine, 5-HT) neurotransmission is regulated by its uptake by the 5-HT transporter (5-HTT), as well as its degradation by monoamine oxidase (MAO)-A. MAO-A deficiency and hypo- methylations lead to a wide set of behavioral alterations, including perseverative behaviors,social deficits and depression(18-21). In addition to that cognitive functions like problem solving were found to be affected by mutations in this gene when it is combined with iron deficiency (ID) in the rhesus monkey $(14,22)$.

Previous evidence of gene-by-environment interactions associated with emotional and behavioral disorders is contradictory. Differences in findings may be related to variation in the dose of the environmental factor, or failure to take account of gene-by-gene interactions (23).

lastly, the studies of the association between MAOAgenes with childhood maltreatment to the development of antisocial behaviors have been replicated, but not consistently. Several methodological issues can justify these discrepancies, including diverse sample, population genetic heterogeneity and exposure to the various adverse environmental factors (24-26). The aim of this study is to analyze the missense single nucleotides polymorphisms in MAOA gene that could result in the occurrence of antisocial and violent behaviors. Up to our knowledge, this will be the first bioinformatics study to address this topic.

\section{Material and Methods:}

\subsection{Retrieving nsSNPs:}

SNPs associated with MAOA gene were obtained from the Single Nucleotide Polymorphism database (dbSNP) in the National Center for Biotechnology Information (NCBI). (http://www.ncbi.nlm.nih.gov/snp/).

The sequence and natural variants of MAOA protein were obtained from the UniProt database as it considered as the most reliable database for protein sequences. (27) (https://www.uniprot.org/).

\subsection{Identifying the most damaging nsSNPs and disease related mutations:}

A total number of 342 nonsynonymous Single Nucleotide Polymorphisms (ns SNPs) were found from the NCBI database, all of them were subjected to in silico analysis using nine different algorithms and softwares; SIFT, PROVEAN, PolyPhen-2, SNPs\&GO, PhD-SNP, PMUT, Imutant, GeneMANIA and Chimera. 


\subsubsection{SIFT Server:}

Phenotypic effects of amino acid substitution on protein function were predicted by using Sorting Intolerant From Tolerant (SIFT) server, which is a powerful tool that uses sequence homology. A list of nsSNPs (rsIDs) from NCBI's dbSNP database were submitted along with the (original) sequence to SIFT to predict tolerated and deleterious substitutions for every position of a protein sequence. The server divides the results into "Deleterious" and "Tolerated", nsSNPs with SIFT score $\leq 0.05$ were classified as deleterious and are further analyzed to identify the damaging ones, and those $>0.05$ were classified as tolerated and are not further analyzed. The server provides fast results but can not assess the result for SNPs that share the same position at the same time. (Available at: http://sift.bii.a-star.edu.sg/)(28-31).

\subsubsection{Provean Server:}

(Protein Variation Effect Analyzer) is the second software tool used. It predicts the effect of an amino acid substitution on the biological function of a protein. It can predict single amino acid substitutions, in-frame insertions, deletions, and multiple amino acid substitutions. The results are obtained as either "Deleterious" if the prediction score was $\leq-2.5$, while score $>-2.5$ indicates that the variant is predicted to have a "Neutral" effect. (Available at: http://provean.jcvi.org/index.php)(32, 33).

\subsubsection{Polyphen-2 Server:}

Polymorphism Phenotyping v2.0 (PolyPhen-2) is another online tool that predicts the possible effects of an amino acid substitution on the structure and function of the protein by analysis of multiple sequence alignment. The results are classified into "probably damaging" that is the most disease causing with a score near to 1 (0.7-1), "possibly damaging" with a less disease causing ability with a score of $0.5-0.8$ and "benign" which does not alter protein functions with a score closer to zero; (0-0.4). We used a patch query as it provide fast and accurate way to get the result. (Available at: http://genetics.bwh.harvard.edu/pph2/)(27, 34, 35).

\subsubsection{SNAP2}

It is another tool to predict the Functional effects of mutations. SNAP2 is a trained classifier that is based on a machine learning device called "neural network". After entering the protein FASTA sequence as an input query, It can distinguish between effect and neutral variants/nonsynonymous SNPs by taking a variety of sequences and variant features into account. The most important input signal for the prediction is the evolutionary information taken from an automatically generated multiple sequence alignment. Also structural features such as predicted 
secondary structure and solvent accessibility are considered. Although it takes a longer time than the previous software to get the result, it has a significant accuracy level ( a sustained two-state accuracy (effect/neutral) of 82\%)(36). It is available at https://rostlab.org/services/snap2web/.

\subsubsection{SNPs \& Go server:}

An online web server that used to ensure the disease relationship with the studied single nucleotide polymorphisms SNPs. It gives three different results based on three different analytical algorithms; Panther result, PHD-SNP result, SNPs\&GO result. Each one of these results is composed of three parts, the prediction which decides whether the mutation is neutral or disease related, reliability index (RI), and disease probability (if $>0.5$ mutations are considered as disease causing nsSNP). (Available at: http://snps-and-go.biocomp.unibo. it/snps-and-go/) (37).

\subsubsection{PMUT Server:}

Is a neural network dependent web-based tool used for the prediction of pathological variants on proteins. The prediction results are classified as "Neutral" or "Disease". It is very fast and accurate, It is available at (http://mmb.irbbarcelona.org/PMut) (38).

\subsection{GeneMANIA:}

A user-friendly web interface tool designed to asses protein function, analyzing submitted gene lists and prioritizing genes for functional assays. It provides lists with functionally similar genes that it identifies and recognizes using available genomics and proteomics data. It is an accurate method to study gene -gene interaction. (Available at: (http://www.genemania.org/)(39).

\subsection{Modeling nsSNP locations on protein structure:}

\subsubsection{Hope project:}

It is a webserver that used to analyze the effect of single point mutation on the structural level of protein. it best way to visualize the mutation since it is to create a report consist of figures,animation, $3 \mathrm{~d}$ structure and mutation report just by submitting the protein sequence and mutation (40). It is available at http://www.cmbi.ru.nl/hope/.

\subsubsection{Chimera:}


Chimera 1.8 software is used for visualization and editing of the three dimensional structure of the protein. It visualizes the original amino acid with the mutated one to see the impact that can be produced. It is a highly extensible program for interactive visualization and analysis of molecular structures and related data, including density maps, supramolecular assemblies,trajectories, and conformational ensembles. High-quality images and animations can be generated. (Availabe at: http://www.cgl.ucsf.edu/chimera)(41).

\section{ConSurf server:}

It is a web server that suggests evolutionary conservation reviews for proteins of known structure in the PDB. ConSurf detects similar amino acid sequences and runs multi alignment approaches. The conserved amino acid across species detects its position using specific algorisms (42).

4. Variant Effect Predictor (VEP):

The Ensembl Variant Effect Predictor software provides toolsets for an organized approach to annotate and assist for the prioritization of mutations. The input data format was a list of variant identifiers while the output was filtered by choosing 1000 genome combined population to expend the population coverage (43).

\section{Result:}

Our results of this study were deduced from analysis of 566 SNPs related to MAOA gene which downloaded from NCBI, 174 SNPs of them were observed as missense mutation. These SNPs were further analyzed by functional and structural softwares to detect the disease associated mutant.

38 SNPs out of 174 SNPs were predicted to be (affect, deleterious, probably damaging, effect) ; after anlysed by SIFT, PolyPhen-2, PROVEAN, SNAP2 soft wares which used to detect the fuctional and structural change on the protein.(Table 1). In addition 6 SNPs out of the 38 SNPs prognosticated to be (disease)when analysed by SNP\&GO, PHD and PMut softwares; it function to ensure disease relationship(Table 2). Eventually, 18 variants were branched from the 6 disease related SNPs by VEP tool. (Table 3).

Table.1. Deleterious nsSNPs associated variations predicted by SIFT, PolyPhen-2, PROVEAN, SNAP2 soft wares.

\begin{tabular}{|c|c|c|c|c|c|c|c|c|c|}
\hline dbSNP rs\# & Sub & $\begin{array}{l}\text { SIFT } \\
\text { PRE }\end{array}$ & $\begin{array}{l}\text { SIFT } \\
\text { SCO } \\
\text { RE }\end{array}$ & $\begin{array}{l}\text { PROVE } \\
\text { EN } \\
\text { Predicti } \\
\text { on } \\
\text { (cutoff= } \\
-2.5)\end{array}$ & $\begin{array}{l}\text { PRO } \\
\text { VEA } \\
\mathrm{N} \\
\text { score }\end{array}$ & $\begin{array}{l}\text { PolyPhen- } \\
22 \\
\text { prediction }\end{array}$ & $\begin{array}{l}\text { pph2_pr } \\
\text { ob }\end{array}$ & $\begin{array}{l}\text { SNAP2 } \\
\text { predicti } \\
\text { on }\end{array}$ & $\begin{array}{l}\text { SNAP2 } \\
\text { score }\end{array}$ \\
\hline rs1268810174 & D15N & $\begin{array}{l}\text { AFFE } \\
\text { CT }\end{array}$ & 0 & $\begin{array}{l}\text { Deleteri } \\
\text { ous }\end{array}$ & -3.01 & $\begin{array}{l}\text { probably } \\
\text { damaging }\end{array}$ & 0.999 & Effect & 90 \\
\hline
\end{tabular}




\begin{tabular}{|c|c|c|c|c|c|c|c|c|c|}
\hline rs1131691720 & L32S & $\begin{array}{l}\text { AFFE } \\
\text { CT }\end{array}$ & 0 & $\begin{array}{l}\text { Deleteri } \\
\text { ous }\end{array}$ & -5.02 & $\begin{array}{l}\text { probably } \\
\text { damaging }\end{array}$ & 1 & Effect & 30 \\
\hline rs796065312 & $\begin{array}{l}\text { R45 } \\
\text { W }\end{array}$ & $\begin{array}{l}\text { AFFE } \\
\text { CT }\end{array}$ & 0 & $\begin{array}{l}\text { Deleteri } \\
\text { ous }\end{array}$ & -6.71 & $\begin{array}{l}\text { probably } \\
\text { damaging }\end{array}$ & 1 & effect & 69 \\
\hline rs201519600 & D46G & $\begin{array}{l}\text { AFFE } \\
\text { CT }\end{array}$ & 0.03 & $\begin{array}{l}\text { Deleteri } \\
\text { ous }\end{array}$ & -5.6 & $\begin{array}{l}\text { possibly } \\
\text { damaging }\end{array}$ & 0.662 & effect & 49 \\
\hline \multirow[t]{2}{*}{ rs750916164 } & $\mathrm{R} 79 \mathrm{C}$ & $\begin{array}{l}\text { AFFE } \\
\text { CT }\end{array}$ & 0.01 & $\begin{array}{l}\text { Deleteri } \\
\text { ous }\end{array}$ & -6.88 & $\begin{array}{l}\text { probably } \\
\text { damaging }\end{array}$ & 1 & effect & 26 \\
\hline & T88I & $\begin{array}{l}\text { AFFE } \\
\text { CT }\end{array}$ & 0.01 & $\begin{array}{l}\text { Deleteri } \\
\text { ous }\end{array}$ & -4.07 & $\begin{array}{l}\text { probably } \\
\text { damaging }\end{array}$ & 0.982 & effect & 34 \\
\hline rs140295792 & $\begin{array}{l}\text { R109 } \\
\text { W }\end{array}$ & $\begin{array}{l}\text { AFFE } \\
\text { CT }\end{array}$ & 0.01 & $\begin{array}{l}\text { Deleteri } \\
\text { ous }\end{array}$ & -3.6 & $\begin{array}{l}\text { probably } \\
\text { damaging }\end{array}$ & 0.997 & effect & 47 \\
\hline rs1157474072 & $\mathrm{I} 138 \mathrm{~F}$ & $\begin{array}{l}\text { AFFE } \\
\text { CT }\end{array}$ & 0.01 & $\begin{array}{l}\text { Deleteri } \\
\text { ous }\end{array}$ & -3.66 & $\begin{array}{l}\text { probably } \\
\text { damaging }\end{array}$ & 0.999 & effect & 7 \\
\hline rs1450929926 & $\begin{array}{l}\text { P139 } \\
\text { Q }\end{array}$ & $\begin{array}{l}\text { AFFE } \\
\text { CT }\end{array}$ & 0.03 & $\begin{array}{l}\text { Deleteri } \\
\text { ous }\end{array}$ & -6.97 & $\begin{array}{l}\text { possibly } \\
\text { damaging }\end{array}$ & 0.904 & effect & 5 \\
\hline rs199524208 & $\begin{array}{l}\text { A146 } \\
\text { V }\end{array}$ & $\begin{array}{l}\text { AFFE } \\
\text { CT }\end{array}$ & 0.03 & $\begin{array}{l}\text { Deleteri } \\
\text { ous }\end{array}$ & -3.07 & $\begin{array}{l}\text { probably } \\
\text { damaging }\end{array}$ & 0.964 & neutral & -12 \\
\hline rs1261565085 & $\begin{array}{l}\text { E159 } \\
\text { K }\end{array}$ & $\begin{array}{l}\text { AFFE } \\
\text { CT }\end{array}$ & 0.05 & $\begin{array}{l}\text { Deleteri } \\
\text { ous }\end{array}$ & -2.88 & $\begin{array}{l}\text { possibly } \\
\text { damaging }\end{array}$ & 0.51 & effect & 51 \\
\hline rs574432879 & $\mathrm{I} 161 \mathrm{~T}$ & $\begin{array}{l}\text { AFFE } \\
\text { CT }\end{array}$ & 0.02 & $\begin{array}{l}\text { Deleteri } \\
\text { ous }\end{array}$ & -3.32 & $\begin{array}{l}\text { possibly } \\
\text { damaging }\end{array}$ & 0.782 & neutral & -56 \\
\hline rs1390855194 & $\begin{array}{l}\text { R172 } \\
\text { W }\end{array}$ & $\begin{array}{l}\text { AFFE } \\
\text { CT }\end{array}$ & 0.01 & $\begin{array}{l}\text { Deleteri } \\
\text { ous }\end{array}$ & -4.13 & $\begin{array}{l}\text { probably } \\
\text { damaging }\end{array}$ & 0.992 & effect & 55 \\
\hline rs77698881 & $\begin{array}{l}\text { E188 } \\
\text { K }\end{array}$ & $\begin{array}{l}\text { AFFE } \\
\text { CT }\end{array}$ & 0.02 & $\begin{array}{l}\text { Deleteri } \\
\text { ous }\end{array}$ & -2.99 & $\begin{array}{l}\text { possibly } \\
\text { damaging }\end{array}$ & 0.804 & effect & 94 \\
\hline rs762184021 & $\begin{array}{l}\text { R217 } \\
\text { W }\end{array}$ & $\begin{array}{l}\text { AFFE } \\
\text { CT }\end{array}$ & 0.01 & $\begin{array}{l}\text { Deleteri } \\
\text { ous }\end{array}$ & -6.68 & $\begin{array}{l}\text { probably } \\
\text { damaging }\end{array}$ & 1 & effect & 65 \\
\hline rs750174700 & $\begin{array}{l}\text { G221 } \\
\text { A }\end{array}$ & $\begin{array}{l}\text { AFFE } \\
\text { CT }\end{array}$ & 0 & $\begin{array}{l}\text { Deleteri } \\
\text { ous }\end{array}$ & -5.66 & $\begin{array}{l}\text { probably } \\
\text { damaging }\end{array}$ & 1 & effect & 62 \\
\hline rs1181634890 & $\begin{array}{l}\text { Q225 } \\
\text { R }\end{array}$ & $\begin{array}{l}\text { AFFE } \\
\text { CT }\end{array}$ & 0.03 & $\begin{array}{l}\text { Deleteri } \\
\text { ous }\end{array}$ & -3.43 & $\begin{array}{l}\text { possibly } \\
\text { damaging }\end{array}$ & 0.827 & effect & 28 \\
\hline \multirow[t]{2}{*}{ rs779805250 } & $\begin{array}{l}\text { R229 } \\
\text { W }\end{array}$ & $\begin{array}{l}\text { AFFE } \\
\text { CT }\end{array}$ & 0.02 & $\begin{array}{l}\text { Deleteri } \\
\text { ous }\end{array}$ & -4.72 & $\begin{array}{l}\text { probably } \\
\text { damaging }\end{array}$ & 0.999 & effect & 36 \\
\hline & $\begin{array}{l}\mathrm{G} 235 \\
\mathrm{R}\end{array}$ & $\begin{array}{l}\text { AFFE } \\
\text { CT }\end{array}$ & 0.02 & $\begin{array}{l}\text { Deleteri } \\
\text { ous }\end{array}$ & -5.39 & $\begin{array}{l}\text { probably } \\
\text { damaging }\end{array}$ & 0.999 & neutral & -13 \\
\hline rs1216988286 & $\begin{array}{l}\text { P243 } \\
\text { S }\end{array}$ & $\begin{array}{l}\text { AFFE } \\
\text { CT }\end{array}$ & 0.04 & $\begin{array}{l}\text { Deleteri } \\
\text { ous }\end{array}$ & -6.37 & $\begin{array}{l}\text { probably } \\
\text { damaging }\end{array}$ & 0.999 & neutral & -31 \\
\hline
\end{tabular}




\begin{tabular}{|c|c|c|c|c|c|c|c|c|c|}
\hline rs587777457 & $\begin{array}{l}\mathrm{C} 266 \\
\mathrm{~F}\end{array}$ & $\begin{array}{l}\text { AFFE } \\
\text { CT }\end{array}$ & 0.01 & $\begin{array}{l}\text { Deleteri } \\
\text { ous }\end{array}$ & -2.93 & $\begin{array}{l}\text { probably } \\
\text { damaging }\end{array}$ & 0.96 & effect & 92 \\
\hline & $\begin{array}{l}\text { V269 } \\
\text { L }\end{array}$ & $\begin{array}{l}\text { AFFE } \\
\text { CT }\end{array}$ & 0.03 & $\begin{array}{l}\text { Deleteri } \\
\text { ous }\end{array}$ & -2.82 & $\begin{array}{l}\text { probably } \\
\text { damaging }\end{array}$ & 0.996 & effect & 37 \\
\hline rs1452282305 & $\begin{array}{l}\text { A272 } \\
\text { V }\end{array}$ & $\begin{array}{l}\text { AFFE } \\
\text { CT }\end{array}$ & 0.01 & $\begin{array}{l}\text { Deleteri } \\
\text { ous }\end{array}$ & -3.73 & $\begin{array}{l}\text { probably } \\
\text { damaging }\end{array}$ & 1 & effect & 68 \\
\hline rs749012045 & $\begin{array}{l}\text { P275 } \\
\text { L }\end{array}$ & $\begin{array}{l}\text { AFFE } \\
\text { CT }\end{array}$ & 0 & $\begin{array}{l}\text { Deleteri } \\
\text { ous }\end{array}$ & -6.3 & $\begin{array}{l}\text { probably } \\
\text { damaging }\end{array}$ & 0.993 & neutral & -19 \\
\hline rs780527254 & $\begin{array}{l}\mathrm{L} 277 \\
\text { S }\end{array}$ & $\begin{array}{l}\text { AFFE } \\
\text { CT }\end{array}$ & 0.01 & $\begin{array}{l}\text { Deleteri } \\
\text { ous }\end{array}$ & -5.72 & $\begin{array}{l}\text { probably } \\
\text { damaging }\end{array}$ & 1 & effect & 56 \\
\hline rs780647851 & $\begin{array}{l}\text { R297 } \\
\text { Q }\end{array}$ & $\begin{array}{l}\text { AFFE } \\
\text { CT }\end{array}$ & 0.03 & $\begin{array}{l}\text { Deleteri } \\
\text { ous }\end{array}$ & -3.9 & $\begin{array}{l}\text { probably } \\
\text { damaging }\end{array}$ & 0.999 & effect & 58 \\
\hline rs61730725 & $\begin{array}{l}\mathrm{L} 298 \\
\mathrm{P}\end{array}$ & $\begin{array}{l}\text { AFFE } \\
\text { CT }\end{array}$ & 0.02 & $\begin{array}{l}\text { Deleteri } \\
\text { ous }\end{array}$ & -4.56 & $\begin{array}{l}\text { probably } \\
\text { damaging }\end{array}$ & 1 & effect & 77 \\
\hline rs867883669 & $\begin{array}{l}\text { P299 } \\
\text { S }\end{array}$ & $\begin{array}{l}\text { AFFE } \\
\text { CT }\end{array}$ & 0.02 & $\begin{array}{l}\text { Deleteri } \\
\text { ous }\end{array}$ & -7.8 & $\begin{array}{l}\text { probably } \\
\text { damaging }\end{array}$ & 1 & effect & 22 \\
\hline rs1799835 & $\begin{array}{l}\text { F314 } \\
\text { V }\end{array}$ & $\begin{array}{l}\text { AFFE } \\
\text { CT }\end{array}$ & 0 & $\begin{array}{l}\text { Deleteri } \\
\text { ous }\end{array}$ & -6.83 & $\begin{array}{l}\text { probably } \\
\text { damaging }\end{array}$ & 1 & effect & 89 \\
\hline rs1451367524 & $\begin{array}{l}\text { P342 } \\
\text { Q }\end{array}$ & $\begin{array}{l}\text { AFFE } \\
\text { CT }\end{array}$ & 0.05 & $\begin{array}{l}\text { Deleteri } \\
\text { ous }\end{array}$ & -7.13 & $\begin{array}{l}\text { probably } \\
\text { damaging }\end{array}$ & 1 & effect & 43 \\
\hline rs1182312989 & $\begin{array}{l}\text { R356 } \\
W\end{array}$ & $\begin{array}{l}\text { AFFE } \\
\text { CT }\end{array}$ & 0.01 & $\begin{array}{l}\text { Deleteri } \\
\text { ous }\end{array}$ & -4.92 & $\begin{array}{l}\text { probably } \\
\text { damaging }\end{array}$ & 0.999 & effect & 56 \\
\hline rs1428368525 & $\begin{array}{l}\text { A378 } \\
\mathrm{T}\end{array}$ & $\begin{array}{l}\text { AFFE } \\
\text { CT }\end{array}$ & 0.01 & $\begin{array}{l}\text { Deleteri } \\
\text { ous }\end{array}$ & -3.16 & $\begin{array}{l}\text { probably } \\
\text { damaging }\end{array}$ & 0.978 & effect & 10 \\
\hline rs1434727379 & T417I & $\begin{array}{l}\text { AFFE } \\
\text { CT }\end{array}$ & 0.01 & $\begin{array}{l}\text { Deleteri } \\
\text { ous }\end{array}$ & -5.23 & $\begin{array}{l}\text { probably } \\
\text { damaging }\end{array}$ & 0.998 & effect & 38 \\
\hline rs1181885385 & $\begin{array}{l}\text { A433 } \\
\mathrm{T}\end{array}$ & $\begin{array}{l}\text { AFFE } \\
\text { CT }\end{array}$ & 0 & $\begin{array}{l}\text { Deleteri } \\
\text { ous }\end{array}$ & -3.77 & $\begin{array}{l}\text { probably } \\
\text { damaging }\end{array}$ & 1 & effect & 21 \\
\hline rs770610370 & $\begin{array}{l}\text { A433 } \\
\text { V }\end{array}$ & $\begin{array}{l}\text { AFFE } \\
\text { CT }\end{array}$ & 0 & $\begin{array}{l}\text { Deleteri } \\
\text { ous }\end{array}$ & -3.77 & $\begin{array}{l}\text { probably } \\
\text { damaging }\end{array}$ & 1 & neutral & -3 \\
\hline rs764516141 & $\begin{array}{l}\text { T439 } \\
\text { R }\end{array}$ & $\begin{array}{l}\text { AFFE } \\
\text { CT }\end{array}$ & 0.03 & $\begin{array}{l}\text { Deleteri } \\
\text { ous }\end{array}$ & -4.87 & $\begin{array}{l}\text { probably } \\
\text { damaging }\end{array}$ & 0.999 & effect & 47 \\
\hline rs1803986 & $\begin{array}{l}\text { M445 } \\
\text { I }\end{array}$ & $\begin{array}{l}\text { AFFE } \\
\text { CT }\end{array}$ & 0.03 & $\begin{array}{l}\text { Deleteri } \\
\text { ous }\end{array}$ & -3.5 & $\begin{array}{l}\text { possibly } \\
\text { damaging }\end{array}$ & 0.925 & neutral & -46 \\
\hline rs1359637885 & $\begin{array}{l}\text { G447 } \\
\text { R }\end{array}$ & $\begin{array}{l}\text { AFFE } \\
\text { CT }\end{array}$ & 0 & $\begin{array}{l}\text { Deleteri } \\
\text { ous }\end{array}$ & -7.54 & $\begin{array}{l}\text { probably } \\
\text { damaging }\end{array}$ & 1 & effect & 93 \\
\hline rs775232342 & $\begin{array}{l}\text { G464 } \\
\text { R }\end{array}$ & $\begin{array}{l}\text { AFFE } \\
\text { CT }\end{array}$ & 0 & $\begin{array}{l}\text { Deleteri } \\
\text { ous }\end{array}$ & -5.27 & $\begin{array}{l}\text { possibly } \\
\text { damaging }\end{array}$ & 0.529 & effect & 3 \\
\hline
\end{tabular}




\begin{tabular}{|c|c|c|c|c|c|c|c|c|}
\hline rs759671679 & $\begin{array}{l}\text { W472 } \\
\mathrm{L}\end{array}$ & $\begin{array}{l}\text { AFFE } \\
\text { CT }\end{array}$ & 0.03 & $\begin{array}{l}\text { Deleteri } \\
\text { ous }\end{array}$ & -9.44 & $\begin{array}{l}\text { possibly } \\
\text { damaging }\end{array}$ & 0.53 & effect \\
\hline rs1254032613 & $\begin{array}{l}\text { R526 } \\
W\end{array}$ & $\begin{array}{l}\text { AFFE } \\
\text { CT }\end{array}$ & 0 & $\begin{array}{l}\text { Deleteri } \\
\text { ous }\end{array}$ & -3.21 & $\begin{array}{l}\text { probably } \\
\text { damaging }\end{array}$ & 0.998 & effect \\
\hline
\end{tabular}

Table. 2. Disease effect nsSNPs associated variations predicted by Pmut, SNP\&GO, PHD and PMut softwares.

\begin{tabular}{|c|c|c|c|c|c|c|c|c|c|}
\hline dbSNP rs\# & sub & $\begin{array}{l}\text { Predictio } \\
\text { n phd }\end{array}$ & $\begin{array}{l}\mathbf{R} \\
\mathbf{I}\end{array}$ & $\begin{array}{l}\text { Probabilit } \\
\text { y }\end{array}$ & $\begin{array}{l}\text { Predictio } \\
\text { n snp } \\
\text { and go }\end{array}$ & $\begin{array}{l}\mathbf{R} \\
\mathbf{I}\end{array}$ & $\begin{array}{l}\text { Probabilit } \\
\text { y }\end{array}$ & $\begin{array}{l}\text { Pmut } \\
\text { predictio } \\
\text { n }\end{array}$ & $\begin{array}{l}\text { Pmu } \\
\mathbf{t} \\
\text { score }\end{array}$ \\
\hline $\begin{array}{l}\text { rs113169172 } \\
0\end{array}$ & L32S & Disease & 7 & 0.87 & Disease & 4 & 0.725 & Disease & $\begin{array}{l}0.90 \\
(93 \% \\
)\end{array}$ \\
\hline rs796065312 & $\mathrm{R} 45 \mathrm{~W}$ & Disease & 8 & 0.921 & Disease & 6 & 0.816 & Disease & $\begin{array}{l}0.90 \\
(93 \% \\
)\end{array}$ \\
\hline rs750174700 & $\begin{array}{l}\mathrm{G} 221 \\
\mathrm{~A}\end{array}$ & Disease & 4 & 0.679 & Disease & 2 & 0.581 & Disease & $\begin{array}{l}0.72 \\
(86 \% \\
)\end{array}$ \\
\hline rs1799835 & $\begin{array}{l}\text { F314 } \\
\text { V }\end{array}$ & Disease & 8 & 0.886 & Disease & 6 & 0.781 & Disease & $\begin{array}{l}0.75 \\
(88 \% \\
)\end{array}$ \\
\hline $\begin{array}{l}\text { rs118188538 } \\
5\end{array}$ & $\begin{array}{l}\text { A433 } \\
\mathrm{T}\end{array}$ & Disease & 4 & 0.717 & Disease & 1 & 0.527 & Disease & $\begin{array}{l}0.83 \\
(90 \% \\
)\end{array}$ \\
\hline $\begin{array}{l}\text { rs135963788 } \\
5\end{array}$ & $\begin{array}{l}\text { G447 } \\
\mathrm{R}\end{array}$ & Disease & 5 & 0.756 & Disease & 4 & 0.717 & Disease & $\begin{array}{l}0.92 \\
(94 \% \\
)\end{array}$ \\
\hline
\end{tabular}

Table (3) Shows variant consequences, transcripts, and regulatory features by VEP tool:

\begin{tabular}{lllllll}
\hline $\begin{array}{l}\text { Uploaded } \\
\text { variation }\end{array}$ & $\begin{array}{l}\text { Alle } \\
\text { le }\end{array}$ & Consequence & IMPACT & $\begin{array}{l}\text { SYMB } \\
\text { OL }\end{array}$ & $\begin{array}{l}\text { Protein } \\
\text { position }\end{array}$ & $\begin{array}{l}\text { Amino } \\
\text { acids }\end{array}$ \\
\hline rs1131691720 & $\mathrm{C}$ & missense variant & $\begin{array}{l}\text { MODER } \\
\text { ATE }\end{array}$ & MAOA & 32 & L/S \\
rs1131691720 & $\mathrm{C}$ & $\begin{array}{l}\text { non coding transcript } \\
\text { exon variant }\end{array}$ & $\begin{array}{l}\text { MODIFI } \\
\text { ER }\end{array}$ & MAOA & - & - \\
rs1131691720 & $\mathrm{C}$ & 5 prime UTR variant & $\begin{array}{l}\text { MODIFI } \\
\text { ER }\end{array}$ & MAOA & - & - \\
rs796065312 & T & missense variant & MODER & MAOA & 45 & R/W
\end{tabular}




\begin{tabular}{|c|c|c|c|c|c|c|}
\hline & & & ATE & & & \\
\hline rs796065312 & $\mathrm{T}$ & $\begin{array}{l}\text { non coding transcript } \\
\text { exon variant }\end{array}$ & $\begin{array}{l}\text { MODIFI } \\
\text { ER }\end{array}$ & MAOA & - & - \\
\hline rs796065312 & $\mathrm{T}$ & 5 prime UTR variant & $\begin{array}{l}\text { MODIFI } \\
\text { ER }\end{array}$ & MAOA & - & - \\
\hline rs750174700 & $\mathrm{C}$ & missense variant & $\begin{array}{l}\text { MODER } \\
\text { ATE }\end{array}$ & MAOA & 221 & $\mathrm{G} / \mathrm{A}$ \\
\hline rs750174700 & $\mathrm{C}$ & $\begin{array}{l}\text { non coding transcript } \\
\text { exon variant }\end{array}$ & $\begin{array}{l}\text { MODIFI } \\
\text { ER }\end{array}$ & MAOA & - & - \\
\hline rs750174700 & $\mathrm{C}$ & missense variant & $\begin{array}{l}\text { MODER } \\
\text { ATE }\end{array}$ & MAOA & 88 & $\mathrm{G} / \mathrm{A}$ \\
\hline rs1799835 & $\mathrm{G}$ & missense variant & $\begin{array}{l}\text { MODER } \\
\text { ATE }\end{array}$ & MAOA & 314 & $\mathrm{~F} / \mathrm{V}$ \\
\hline rs1799835 & $\mathrm{G}$ & downstream gene variant & $\begin{array}{l}\text { MODIFI } \\
\text { ER }\end{array}$ & MAOA & - & - \\
\hline rs1799835 & $\mathrm{G}$ & missense variant & $\begin{array}{l}\text { MODER } \\
\text { ATE }\end{array}$ & MAOA & 181 & $\mathrm{~F} / \mathrm{V}$ \\
\hline rs1181885385 & A & missense variant & $\begin{array}{l}\text { MODER } \\
\text { ATE }\end{array}$ & MAOA & 433 & $\mathrm{~A} / \mathrm{T}$ \\
\hline rs1181885385 & A & $\begin{array}{l}\text { non coding transcript } \\
\text { exon variant }\end{array}$ & $\begin{array}{l}\text { MODIFI } \\
\text { ER }\end{array}$ & MAOA & - & - \\
\hline rs1181885385 & A & missense variant & $\begin{array}{l}\text { MODER } \\
\text { ATE }\end{array}$ & MAOA & 300 & $\mathrm{~A} / \mathrm{T}$ \\
\hline rs1359637885 & A & missense variant & $\begin{array}{l}\text { MODER } \\
\text { ATE }\end{array}$ & MAOA & 447 & $\mathrm{G} / \mathrm{R}$ \\
\hline rs1359637885 & A & $\begin{array}{l}\text { non coding transcript } \\
\text { exon variant }\end{array}$ & $\begin{array}{l}\text { MODIFI } \\
\text { ER }\end{array}$ & MAOA & - & - \\
\hline rs1359637885 & A & missense variant & $\begin{array}{l}\text { MODER } \\
\text { ATE }\end{array}$ & MAOA & 314 & $\mathrm{G} / \mathrm{R}$ \\
\hline
\end{tabular}

The reference sequence was submitted to RaptorX, to get the structural prediction of the protein, which was further displayed by Chimera software; to visualize the changes in the wild and mutant amino acids.(Figure 1-6). Then gene to gene relations was identified using geneMAINA 
software (Figure7).moreover, the conservation of the SNPs among different species was detected using ConSurf software (Figure8).

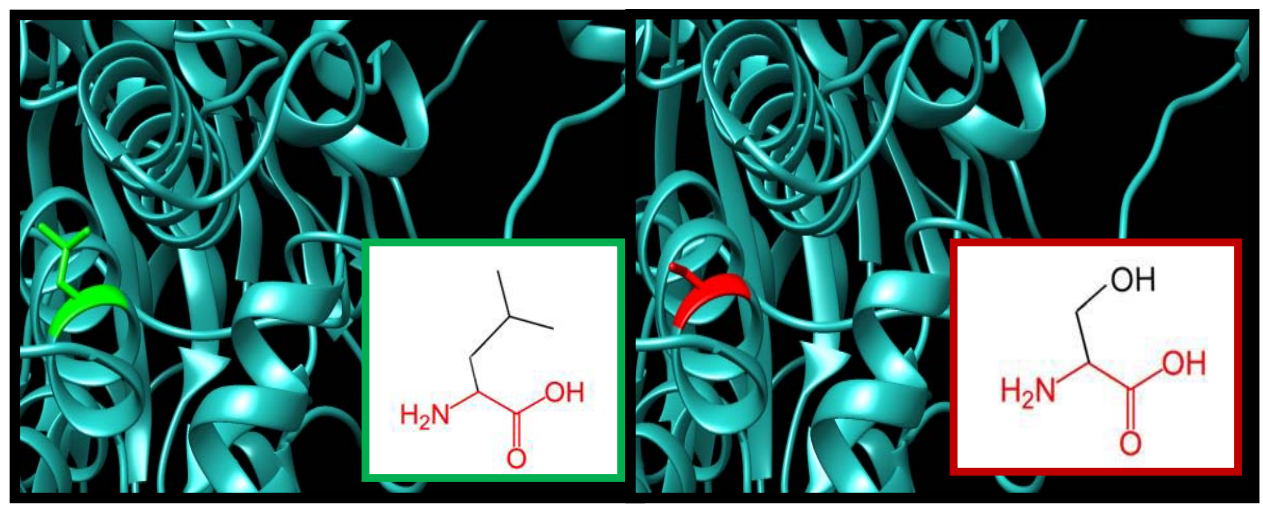

Figure 1:L32S: Leucine (green color) changed to Serine (red color) at position 32. The green color represents wild amino acid while the red color represents mutant amino acid and the cyan color represents the background structure of the MAOA protein.

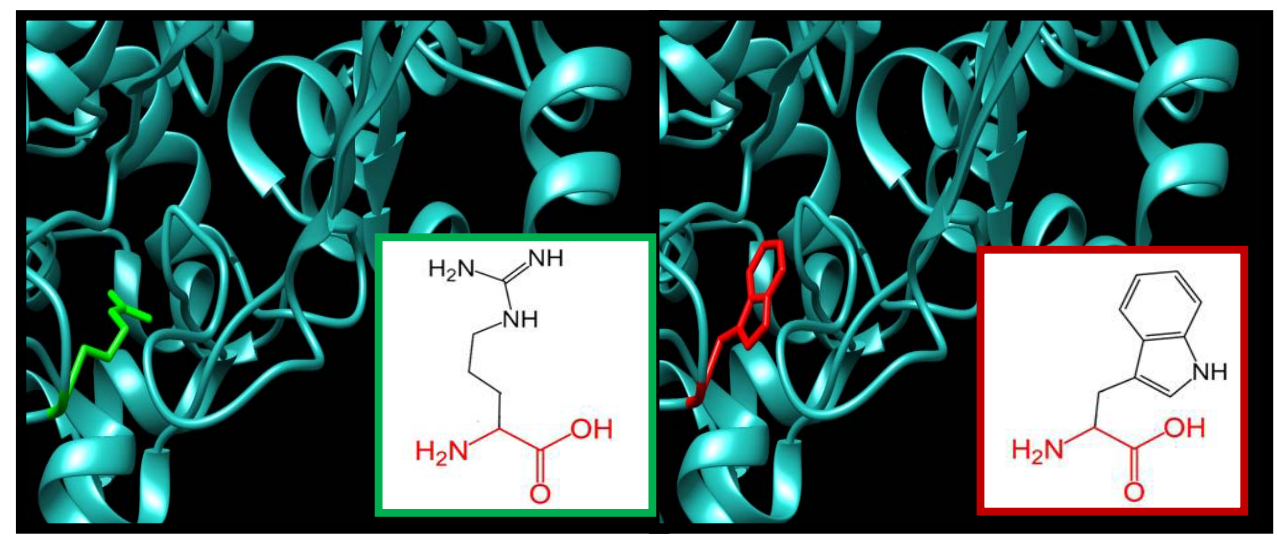

Figure 2:A45W: Arginine(green color) changed to Tryptophan (red color) at position 45. The green color represents wild amino acid while the red color represents mutant amino acid and the cyan color represents the background structure of the MAOA protein. 


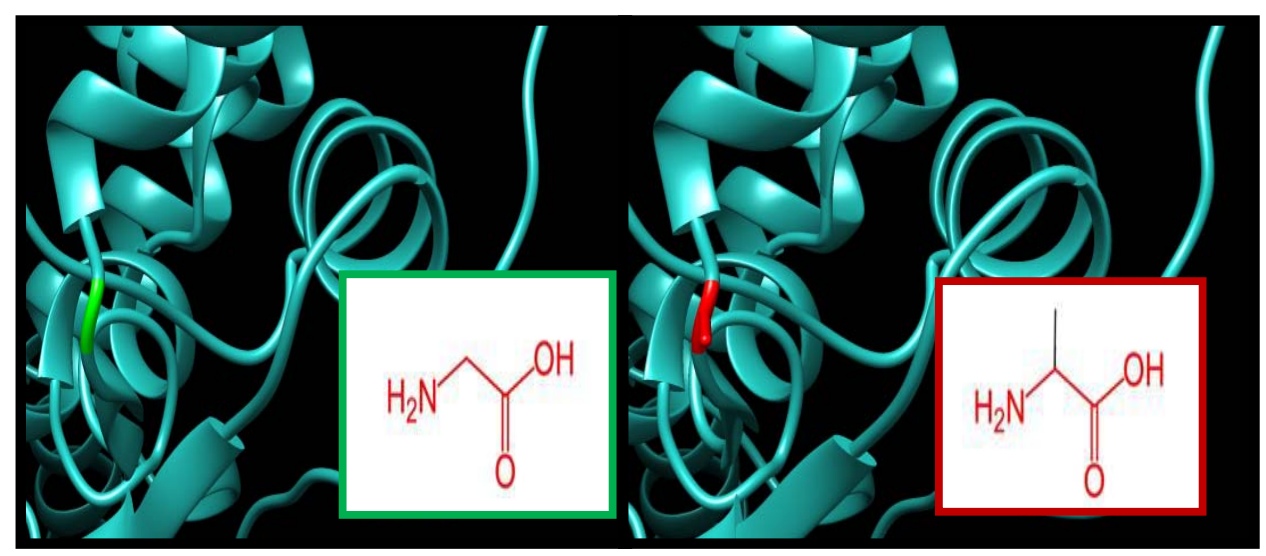

Figure3:G221 A:Glycine (green color) changed to Alanine(red color) at position 221. The green color represents wild amino acid while The red color represents mutant amino acid and the cyan color represents the background structure of the MAOA protein.

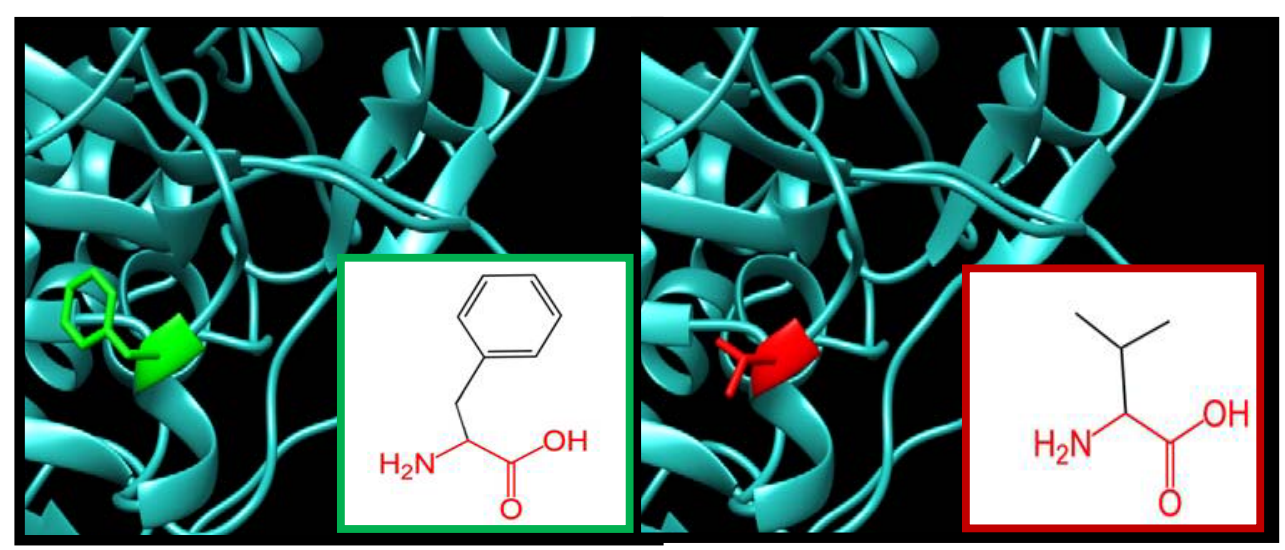

Figure4:F314V:Phenylalanine (green color) changed to Valine(red color) at position 314. The green color represents wild amino acid while The red color represents mutant amino acid and the cyan color represents the background structure of the MAOA protein. 


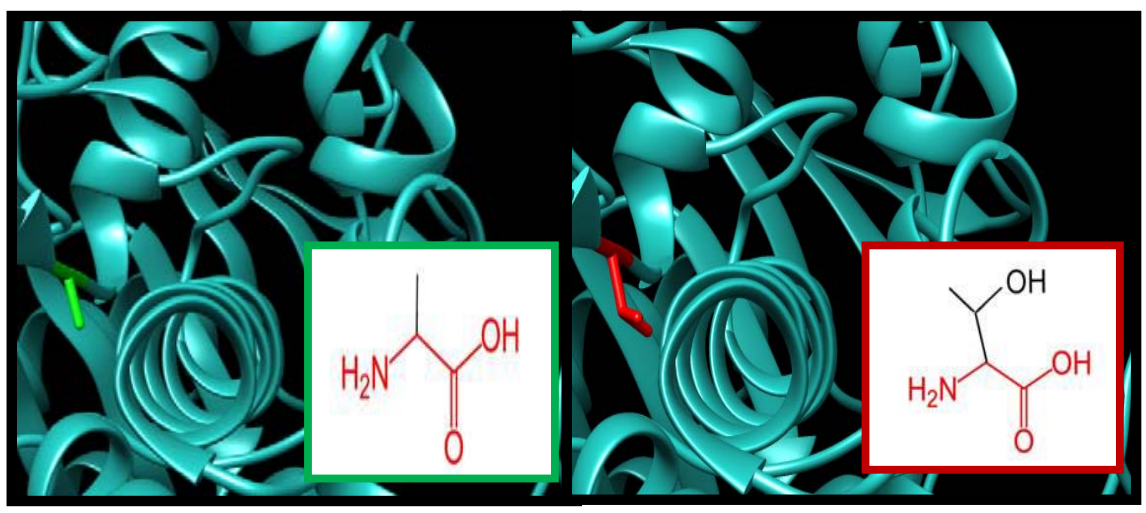

Figure 5:A422T: Alanine(green color) changed to Threonine(red color) at position 433. The green color represents wild amino acid while The red color represents mutant amino acid and the cyan color represents the background structure of the MAOA protein.

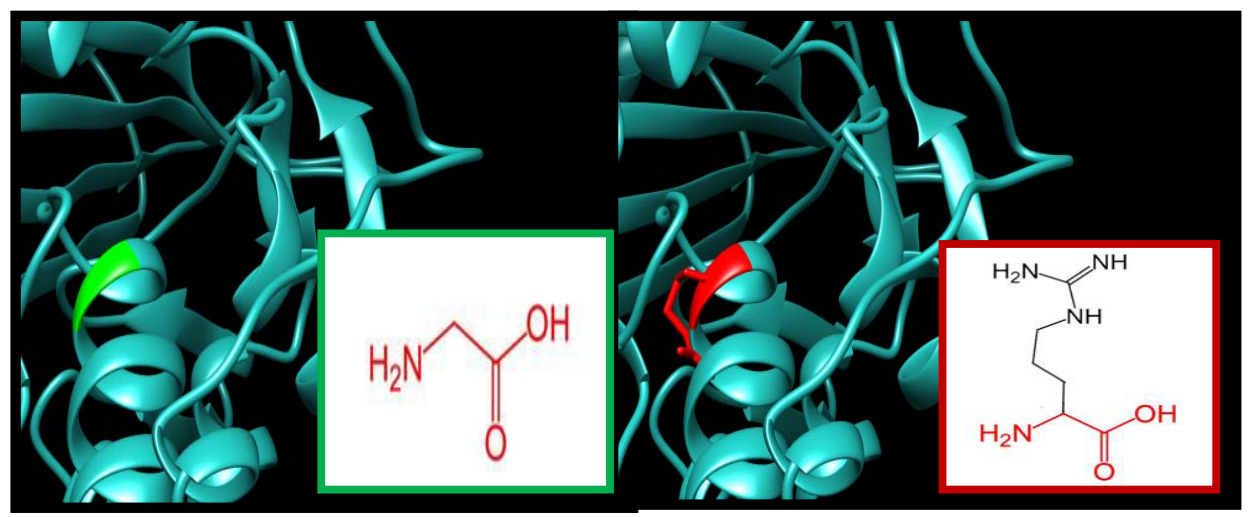

Figure6:G447A: Glycine (green color) changed to Arginine (red color) at position 447. The green color represents wild amino acid while The red color represents mutant amino acid and the cyan color represents the background structure of the MAOA protein. 
bioRxiv preprint doi: https://doi.org/10.1101/2019.12.18.880963; this version posted July 13,2020 . The copyright holder for this preprint (which was not certified by peer review) is the author/funder, who has granted bioRxiv a license to display the preprint in perpetuity. It is made available under aCC-BY-NC-ND 4.0 International license.

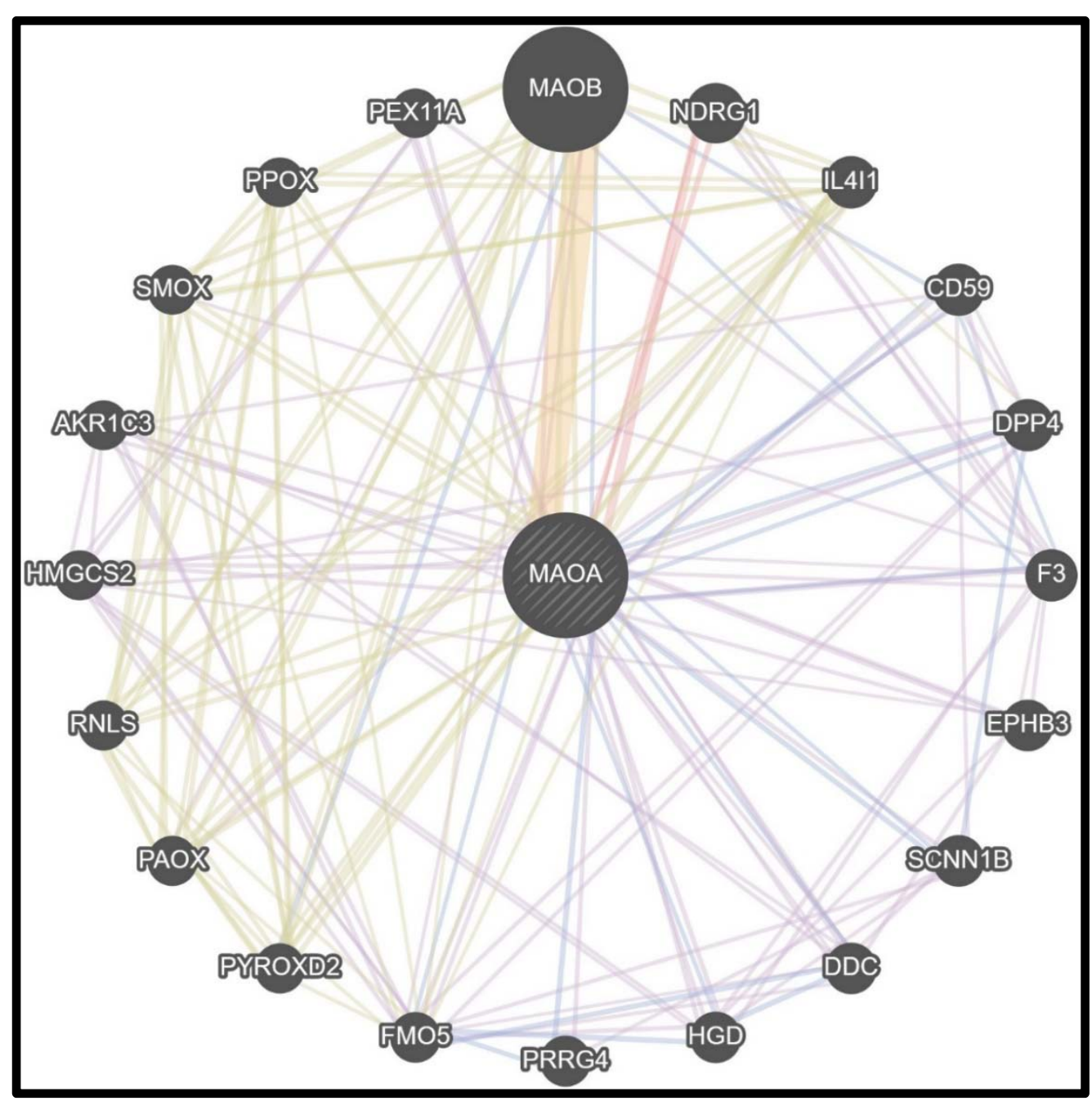

Figure(7): shows gene-gene interaction by geneMANIA. The lines represent the complicated interactions between MAOA gene and other genes. 


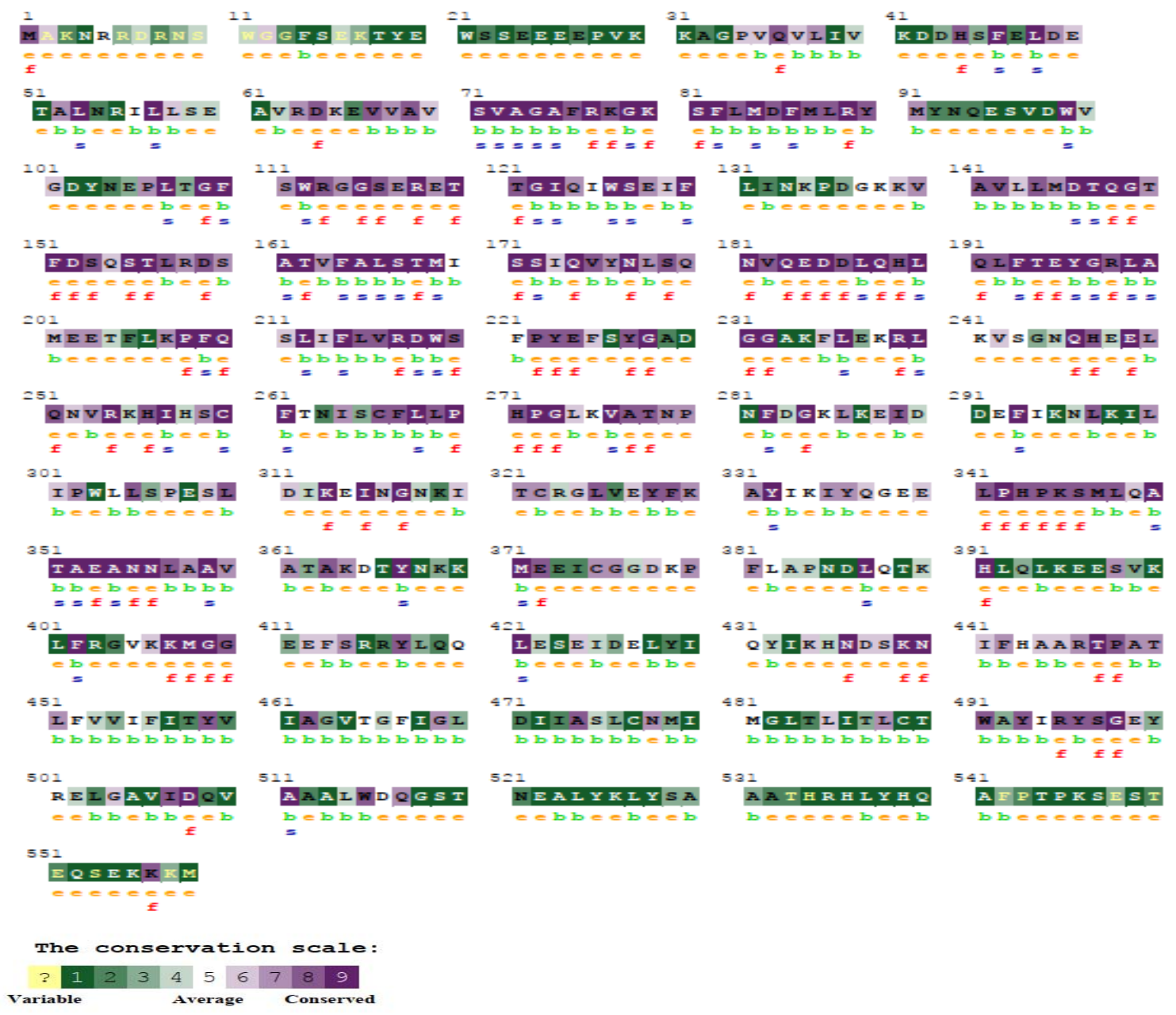

Figure( 8): The conserved amino acids across species in MAOA protein were determined using ConSurf. e: exposed residues according to the neural-network algorithm are indicated in orange letters. b: residues predicted to be buried are demonstrated via green letters. f: predicted functional residues (highly conserved and exposed) are indicated with red letters. s: predicted structural residues (highly conserved and buried) are demonstrated in blue letters. I: insufficient data (the calculation for this site performed in less than $10 \%$ of the sequences) is demonstrated in yellow letters. 


\section{6- Discussion:}

Six novel missense SNPs were found in MAOA gene to effect protein function and structure. Out of 566 SNPS downloaded from NCBI, 34 SNPs have been found to be damaging by four functional software. Which were further analyzed by SNP\& GO and PMut, 6 SNPs were found to be damaging, as illustrated in table (1 and 2).

Project hope was used to study the physiochemical changes inflicted by the missense mutations. All the SNPs were found to be located at Fad/Nad(P)-Binding Domain Superfamily IPR036188 and Flavin Amine Oxidase IPR001613 domain which indicates this SNPs is likely to be conserved. In all of the SNPs the mutant amino acid was bigger in size than the wild type except in F314V and L32S SNPs. In addition all the mutant amino acids were found to be different in hydrophobicity when compared with the wild type amino acid. All these changes will affect protein function.

Structural changes were further studied using chimera software, where changes in the amino acids Cleary change the structural of the MAOA protein as evident in figure( 1-6 ).

The interaction and function of MAOA gene were studied using gene mania. where the predicted functions were: amine metabolic process, cellular amine metabolic process, cellular biogenic amine metabolic process, cellular response to xenobiotic stimulus, oxidoreductase activity, acting on the $\mathrm{CH}-\mathrm{NH} 2$ group of donors, oxidoreductase activity, acting on the $\mathrm{CH}-\mathrm{NH} 2$ group of donors, oxygen as acceptor, response to xenobiotic stimulus and xenobiotic metabolic process. The MAOA gene was found to have interactions with many other genes like CD50,F3,HGD and others as shown in Figure (7).

The Variant Effect Predictor annotates mutations using an extensive array of reference data from previously detected mutations, evidence based results, and estimation of biophysical consequences of mutations; and that is what makes VEP an accurate web based tool. .(43) VEP described regulatory consequences for several mutations, including 10 mutations within the coding region, 5 mutations within a non-coding region. briefly, mutations within coding region affect the protein function, while mutations within non-coding regions can significantly affect disease and could contribute in the phenotypic feature and RNA-binding proteins (RBPs) (44, 45), while mutations in the upstream, downstream, 5'-, and $3^{\prime}$-UTRs might affect transcription or translation process (46). The consequences are shown in Table(3).

We also used ConSurf web server; the nsSNPs that are located in highly conserved regions and predicted to cause structural and functional impacts on MAOA protein Figure (8)

Previous researches show that regulatory variations in MAOA to moderate effects of childhood maltreatment on male antisocial behaviors (26). while another research showed that certain SNPs polymorphism $(1460 \mathrm{C}>\mathrm{T})$ is associated with other conditions leading to the development of depressive symptoms in menopausal women (47). On the other hand another research underwent in Chinese population, the interaction between catechol-O-methyltransferase (COMT), Ala22/72Ser and MAOA T941G polymorphisms, and stressful life events (SLE) in the academic pressure and the relation to aggressive behavior development were also identified (48). This 
SNP was not available in the data we acquired from NCBI.Furthermore, these studies do indicate the significant role of MAOA gene polymorphisms in aggrieve behavior development.

There are other studies that assess the contribution of gene mutations in the mediation of directed aggression in humans, as in the study done on a population of 328 German subjects which reported that, the functional polymorphism in Chatechol-O-methyltranferese (COMT) gene can alter the phenotype of suicidal and anger behaviors(49).

From the previous evidence we believe that a screening for MAOA, COMT and other similar genes mutation in suspected individuals may help in detecting earlier violent behavior in popullations which will provide the chance for earlier intervention, leading to decrease in the incidence of aggressive behavior and criminal act.

As we report in this paper further illustrate the significant role that genetics plays in the development of violent behavior, we still believe that every one has a choice at some point in his life whether to succump to his inner devil or not.

This study is limited, as it does not cover the environmental factor important for the development of antisocial behavior, nevertheless this study will lay the foundation for further clinical research in this phenomenon.

\section{7- Conclusion:}

Six novel missense SNPs were found in MAOA gene that affect MAOA protein function and structure, which could predispose to violent and aggressive behavior. Therefore this gene along with other similar genes colud be used as a surveillance tool for early detection of aggressive behavior in the population.

\section{8- Conflict of interest:}

The authors decline any conflict of interest regarding the making of this paper.

\section{9- Acknowledgment:}

The authors acknowledge the Deanship of Scientific Research at the University of Bahri for supportive cooperation.

\section{0- Funding Statement:}

The authors received no financial support for the research, authorship, and/or publication of this article.

\section{Data Availability:}

All data are available within this manuscript. 


\section{Reference:}

1. DeBono A, Muraven M. Rejection perceptions: Feeling disrespected leads to greater aggression than feeling disliked. Journal of Experimental Social Psychology. 2014;55:43-52.

2. Bell CC. DSM-IV: Diagnostic and Statistical Manual of Mental Disorders. Jama. 1994;272(10):828-

9.

3. Aslund C, Nordquist N, Comasco E, Leppert J, Oreland L, Nilsson KW. Maltreatment, MAOA, and delinquency: sex differences in gene-environment interaction in a large population-based cohort of adolescents. Behav Genet. 2011;41(2):262-72.

4. Personality Disorders. Diagnostic and Statistical Manual of Mental Disorders. DSM Library: American Psychiatric Association; 2013.

5. Jacob CP, Müller J, Schmidt M, Hohenberger K, Gutknecht L, Reif A, et al. Cluster B Personality Disorders are Associated with Allelic Variation of Monoamine Oxidase A Activity.

Neuropsychopharmacology. 2005;30(9):1711-8.

6. Disruptive, Impulse-Control, and Conduct Disorders. Diagnostic and Statistical Manual of Mental Disorders. DSM Library: American Psychiatric Association; 2013.

7. Denson TF, Dobson-Stone C, Ronay R, von Hippel W, Schira MM. A functional polymorphism of the MAOA gene is associated with neural responses to induced anger control. J Cogn Neurosci. 2014;26(7):1418-27.

8. Hwang IW, Lim MH, Kwon HJ, Jin HJ. Association of Monoamine Oxidase A (MAOA) Gene uVNTR and rs6323 Polymorphisms with Attention Deficit and Hyperactivity Disorder in Korean Children. Medicina (Kaunas). 2018;54(3).

9. Klasen M, Wolf D, Eisner PD, Habel U, Repple J, Vernaleken I, et al. Neural networks underlying trait aggression depend on MAOA gene alleles. Brain Struct Funct. 2018;223(2):873-81.

10. McDermott R, Tingley D, Cowden J, Frazzetto G, Johnson DD. Monoamine oxidase A gene (MAOA) predicts behavioral aggression following provocation. Proc Natl Acad Sci U S A. 2009;106(7):2118-23.

11. Beaver KM, Barnes JC, Boutwell BB. The 2-repeat allele of the MAOA gene confers an increased risk for shooting and stabbing behaviors. Psychiatr Q. 2014;85(3):257-65.

12. Craig IW. The role of monoamine oxidase A, MAOA, in the aetiology of antisocial behaviour: the importance of gene-environment interactions. Novartis Found Symp. 2005;268:227-37; discussion 3741, 42-53.

13. Bortolato $M$, Floris $G$, Shih JC. From aggression to autism: new perspectives on the behavioral sequelae of monoamine oxidase deficiency. J Neural Transm (Vienna). 2018;125(11):1589-99.

14. Golub MS, Hogrefe CE, Unger EL. Influence of prenatal iron deficiency and MAOA genotype on response to social challenge in rhesus monkey infants. Genes Brain Behav. 2012;11(3):278-90.

15. Lei $H$, Zhang X, Di X, Rao H, Ming Q, Zhang J, et al. A functional polymorphism of the MAOA gene modulates spontaneous brain activity in pons. Biomed Res Int. 2014;2014:243280.

16. Bortolato $\mathrm{M}$, Shih JC. Behavioral outcomes of monoamine oxidase deficiency: preclinical and clinical evidence. Int Rev Neurobiol. 2011;100:13-42.

17. Edgnulu TG, Ozge A, Erdal N, Kuru O, Erdal ME. Association analysis of the functional MAOA gene promoter and MAOB gene intron 13 polymorphisms in tension type headache patients. Adv Clin Exp Med. 2014;23(6):901-6.

18. Godar SC, Bortolato M, Castelli MP, Casti A, Casu A, Chen K, et al. The aggression and behavioral abnormalities associated with monoamine oxidase $A$ deficiency are rescued by acute inhibition of serotonin reuptake. J Psychiatr Res. 2014;56:1-9.

19. Kim SK, Park HJ, Seok H, Jeon HS, Chung JH, Kang WS, et al. Association study between monoamine oxidase $A(M A O A)$ gene polymorphisms and schizophrenia: lack of association with 
schizophrenia and possible association with affective disturbances of schizophrenia. Mol Biol Rep. 2014;41(5):3457-64.

20. Guo H, Ren Y, Zhao N, Wang Y, Li S, Cui H, et al. Synergistic effect of 5-HT2A receptor gene and MAOA gene on the negative emotion of patients with depression. Clin Physiol Funct Imaging. 2014;34(4):277-81.

21. Schiele MA, Ziegler C, Kollert L, Katzorke A, Schartner C, Busch Y, et al. Plasticity of Functional MAOA Gene Methylation in Acrophobia. Int J Neuropsychopharmacol. 2018;21(9):822-7.

22. Golub M, Hogrefe C. Prenatal iron deficiency and monoamine oxidase A (MAOA) polymorphisms: combined risk for later cognitive performance in rhesus monkeys. Genes Nutr. 2014;9(2):381.

23. Nilsson KW, Comasco E, Hodgins S, Oreland L, Aslund C. Genotypes do not confer risk for delinquency but rather alter susceptibility to positive and negative environmental factors: geneenvironmentinteractions of BDNF Val66Met, 5-HTTLPR, and MAOA-uVNTR [corrected]. Int J Neuropsychopharmacol. 2014;18(5).

24. Ouellet-Morin I, Cote SM, Vitaro F, Hebert M, Carbonneau R, Lacourse E, et al. Effects of the MAOA gene and levels of exposure to violence on antisocial outcomes. Br J Psychiatry. 2016;208(1):42-

8.

25. Pingault JB, Cote SM, Booij L, Ouellet-Morin I, Castellanos-Ryan N, Vitaro F, et al. Age-dependent effect of the MAOA gene on childhood physical aggression. Mol Psychiatry. 2013;18(11):1151-2.

26. Byrd AL, Manuck SB. MAOA, childhood maltreatment, and antisocial behavior: meta-analysis of a gene-environment interaction. Biol Psychiatry. 2014;75(1):9-17.

27. Adzhubei I, Jordan DM, Sunyaev SR. Predicting functional effect of human missense mutations using PolyPhen-2. Curr Protoc Hum Genet. 2013;Chapter 7:Unit7 20.

28. $\mathrm{Ng} \mathrm{PC}$, Henikoff SJGr. Accounting for human polymorphisms predicted to affect protein function. 2002;12(3):436-46.

29. Manickam M, Ravanan $P$, Singh $P$, Talwar P. In silico identification of genetic variants in glucocerebrosidase (GBA) gene involved in Gaucher's disease using multiple software tools. Front Genet. 2014;5:148.

30. Ng PC, Henikoff SJARGHG. Predicting the effects of amino acid substitutions on protein function. 2006;7:61-80.

31. Schneider G, Hu J, Sim N-L, Kumar P, Henikoff S, Ng PC.

32. Choi Y, Sims GE, Murphy S, Miller JR, Chan AP. Predicting the functional effect of amino acid substitutions and indels. PLoS One. 2012;7(10):e46688.

33. Kumar P, Mahalingam K. In silico approach to identify non-synonymous SNPs with highest predicted deleterious effect on protein function in human obesity related gene, neuronal growth regulator 1 (NEGR1). 3 Biotech. 2018;8(11):466.

34. Ma J, Wang $S$, Zhao $F$, Xu J.

35. Adzhubei IA, Schmidt S, Peshkin L, Ramensky VE, Gerasimova A, Bork P, et al. A method and server for predicting damaging missense mutations. Nature methods. 2010;7(4):248-9.

36. Hecht M Fau - Bromberg Y, Bromberg Y Fau - Rost B, Rost B. Better prediction of functional effects for sequence variants. (1471-2164 (Electronic)).

37. Calabrese R, Capriotti E, Fariselli P, Martelli PL, Casadio RJHm. Functional annotations improve the predictive score of human disease-related mutations in proteins. 2009;30(8):1237-44.

38. López-Ferrando V, Gazzo A, de la Cruz X, Orozco M, Gelpí JLJNar. PMut: a web-based tool for the annotation of pathological variants on proteins, 2017 update. 2017;45(W1):W222-W8.

39. Warde-Farley D, Donaldson SL, Comes O, Zuberi K, Badrawi R, Chao P, et al. The GeneMANIA prediction server: biological network integration for gene prioritization and predicting gene function. 2010;38(suppl_2):W214-W20. 
40. Venselaar H, Te Beek Ta Fau - Kuipers RKP, Kuipers Rk Fau - Hekkelman ML, Hekkelman MI Fau Vriend G, Vriend G. Protein structure analysis of mutations causing inheritable diseases. An e-Science approach with life scientist friendly interfaces. (1471-2105 (Electronic)).

41. Pettersen EF, Goddard TD, Huang CC, Couch GS, Greenblatt DM, Meng EC, et al. UCSF Chimeraa visualization system for exploratory research and analysis. 2004;25(13):1605-12.

42. Ashkenazy H, Abadi S, Martz E, Chay O, Mayrose I, Pupko T, et al. ConSurf 2016: an improved methodology to estimate and visualize evolutionary conservation in macromolecules. Nucleic acids research. 2016;44(W1):W344-50.

43. McLaren W, Gil L, Hunt SE, Riat HS, Ritchie GR, Thormann A, et al. The Ensembl Variant Effect Predictor. Genome biology. 2016;17(1):122.

44. Rojano E, Seoane P, Ranea JAG, Perkins JR. Regulatory variants: from detection to predicting impact. Briefings in bioinformatics. 2018.

45. Dhamija S, Menon MB. Non-coding transcript variants of protein-coding genes - what are they good for? RNA biology. 2018;15(8):1025-31.

46. Tatarinova TV, Chekalin E, Nikolsky Y, Bruskin S, Chebotarov D, McNally KL, et al. Nucleotide diversity analysis highlights functionally important genomic regions. Scientific reports. 2016;6:35730.

47. Rozycka A, Slopien R, Slopien A, Dorszewska J, Seremak-Mrozikiewicz A, Lianeri M, et al. The MAOA, COMT, MTHFR and ESR1 gene polymorphisms are associated with the risk of depression in menopausal women. Maturitas. 2016;84:42-54.

48. Wang M, Li H, Deater-Deckard K, Zhang W. Interacting Effect of Catechol-O-Methyltransferase (COMT) and Monoamine Oxidase A (MAOA) Gene Polymorphisms, and Stressful Life Events on Aggressive Behavior in Chinese Male Adolescents. Front Psychol. 2018;9:1079.

49. Rujescu D, Giegling I, Gietl A, Hartmann AM, Möller H-J. A functional single nucleotide polymorphism (V158M) in the COMT gene is associated with aggressive personality traits. Biological Psychiatry. 2003;54(1):34-9. 